\title{
MHD EFFECTS ON NON-NEWTONIAN MICRO POLAR FLUID WITH UNIFORM SUCTION/BLOWING AND HEAT GENERATION IN THE PRESENCE OF CHEMICAL REACTION AND THERMOPHORESIS
}

\author{
N. Kishan ${ }^{1}$ and S. Jagadha ${ }^{2}$ \\ ${ }^{1}$ Department of Mathematics, University College of Science, Osmania University, Hyderabad, A.P., India \\ ${ }^{2}$ Department of Mathematics, Hyderabad Institute of technology and Management, Medchal, Hyderabad, A.P, .India, \\ kishan_n@rediffmail.com, jagadhasaravanan@gmail.com
}

\begin{abstract}
In this present work, the problem of heat and mass transfer on the flow of Non-newtonian micropolar fluid with uniform suction/blowing with heat generation, radiation, thermophoresis and chemical reaction effects are studied. The non-linear partial differential equation arising from the flow modeling were transformed into coupled non linear ordinary differential equations and subsequently solved by using the implicit finite difference method. The effect of relevant thermo physical parameters such as material parameter $K$, suction parameter A, heat generation/absorption parameter B, Prandtl number Pr, radiation parameter $R$, thermophoretic parameter $\tau$, chemical reaction parameter $\delta$, magnetic parameter Ha and Schmidt number Sc are also being numerically investigated and analyzed.
\end{abstract}

Keywords: MHD, micro polar fluid, heat generation, thermophoresis, radiation and finite difference scheme.

\section{INTRODUCTION}

The dynamics of micropolar fluids has attracted considerable attention during the last few decades because traditional Newtonian fluids cannot precisely describe the characteristics of fluid flow with suspended particles. Eringen [1] developed the theory that the local effects arising from the microstructure and the intrinsic motion of the fluid elements should be taken into account. The theory is expected to provide a mathematical model for the Non-Newtonian fluid behavior observed in certain man-made liquids such as polymers, lubricants, fluids with additives, paints, animal blood and colloidal and suspension solutions, etc. The presence of dust or smoke, particularly in a gas, may also be modeled using micropolar fluid dynamics. Later, Eringen [2] extended the theory of thermo-micropolar fluids and derived the constitutive laws for fluids with microstructures. An excellent review of micropolar fluids and their applications was given by Ariman et al. [3], Gorla [4] discussed the steady state heat transfer in a micropolar fluid flow over a semi-infinite plate and the analysis is based on similarity variables. Rees and Pop[5] studied the free convection boundary layer flow of micropolar fluid from a vertical flat plate. Singh [6] has studied the free convection flow of a micropolar fluid past an infinite vertical plate using the finite difference method

Chemical reactions can be codified as either homogeneous or heterogeneous processes. A homogeneous reaction is one that occurs uniformly through a given phase. In contrast, a heterogeneous reaction takes place in a restricted region or within the boundary of a phase. A reaction is said to be the first order if the rate of reaction is directly proportional to the concentration itself. In many chemical engineering processes, a chemical reaction between a foreign mass and the fluid does occur. These processes take place in numerous industrial applications, such as the polymer production, the manufacturing of ceramics or glassware, the food processing [7] and so on. Das et al.[8] considered the effects of a first order chemical reaction on the flow past an impulsively started infinite vertical plate with constant heat flux and mass transfer. Muthucumarswamy and Ganesan [9] and Muthucumarswamy [10] studied the first order homogeneous chemical reaction on the flow past an infinite vertical plate.

\begin{abstract}
Thermophoresis is a mechanism of migration of small particles in direction of decreasing thermal gradient by Hinds.W.C [11]. It is an effective method for particle collection by Sai et al.[12]. The velocity acquired by the particle is called thermophoretic velocity and the force experienced by the suspended particle is called thermophoretic force by Bakier et al. [13]. Thermophoresis causes small particles to deposit on the cold surfaces. It has many applications in aerosol technology, deposition of silicon thin films, and radioactive particle deposition in nuclear reactor safety simulations.
\end{abstract}


Also, the study of micropolar fluids is very significant due to their potential application in many industrial processes; for example, in continuous casting glass-fiber production, paper production, metal extrusion, hot rolling, wire drawing, drawing of plastic films, metal and polymer extrusion and metal spinning. Balaram and Sastry [14] solved the problem of a fully developed free convection flow in a micropolar flow. Agarwal and Dhanapal [15] obtained a numerical solution to study the fully developed free convection micropolar fluid flow between two parallel with constant suction (or injection). Srinivasacharya et al. [16] studied the effects of microrotation and frequency parameters on an unsteady flow of micropolar fluid between two parallel porous plates with a periodic suction.

The interaction of magnetic field and microrotation plays a vital role in several engineering applications such as in MHD electrical power generation, designing cooling system for nuclear reactors, etc., where microrotation provides an important parameter for deciding the rate of heat flow. Gorla et al. [17] developed a numerical scheme to solve the steady free convection from a vertical isothermal plate in a strong cross magnetic field immersed in a micropolar fluid.

El-Hakiem et al. [18] analyzed the effect of viscous and Joule heating on the flow of an electrically conducting and micropolar fluid past a plate whose temperature varies linearly with the distance from the leading edge in the presence of a uniform transverse magnetic field. Helmy et al. [19] studied the unsteady flow MHD of a conducting micropolar fluid, through a porous medium, over an infinite plate that is set in motion in its own plane by an impulse. Bhargava et al. [20] obtained a numerical solution of a free convection MHD micropolar fluid flow between two parallel porous vertical plates by means of the quasi-linearization method.

The study of heat generation/absorption effects in moving fluids is important in view of several physical problems, such as fluids undergoing exothermic or endothermic chemical reaction. Due to the fast growth of electronic technology, effective cooling of electronic equipment has become warranted. The cooling of electronic equipment ranges from individual transistors to main frame computers, from energy suppliers to telephone switch boards and thermal diffusion effects has been utilized for isotopes separation in the mixture between gases with very light molecule weight (hydrogen and helium) and medium molecular weight. Recently R. A. Mohamed1 et al. and Ziabakhsh et al. studied [21.22] Heat and mass transfer analysis on the flow of non-Newtonian micropolar fluid with uniform suction/blowing, heat generation, chemical reaction and Thermophoresis .

The purpose of study is to investigate the effects of MHD on non-Newtonian micro polar fluid flow with uniform suction/blowing heat generation, radiation, thermophoresis and chemical reaction. We solved the non-linear boundary value problem arising from the non dimensionalization and local non similarity method using an implicit finite difference scheme along with Gauss-Seidal method. The Cprogramming code is used to solve the system equations.

\section{MATHEMATICAL FORMULATION}

Consider the two-dimensional stagnation point flow of an incompressible non-Newtonian micropolar fluid implinging perpendicular on a permeable wall and flowing away along the $\mathrm{x}$-axis. A uniform magnetic field $\beta \mathrm{o}$ is applied normal to the walls. And using the boundary layer approximation and neglecting the dissipation, the equation of energy for temperature $\mathrm{T}$ with heat generation or absorption and thermal radiation, the equation of mass for concentration $\mathrm{C}$ with thermophoresis and chemical reaction. The simplified twodimensional equations governing the flow in the boundary layer of a steady, laminar and incompressible micropolar fluid are governed by:

$\frac{\partial \mathrm{u}}{\partial \mathrm{x}}+\frac{\partial \mathrm{v}}{\partial \mathrm{y}}$

$\rho\left(\mathrm{u} \frac{\partial \mathrm{u}}{\partial \mathrm{x}}+\mathrm{v} \frac{\partial \mathrm{v}}{\partial \mathrm{y}}\right)=(\mu+\mathrm{k})\left(\frac{\partial^{2} \mathrm{u}}{\partial \mathrm{y}^{2}}\right)+\mathrm{k} \frac{\partial \mathrm{N}}{\partial \mathrm{y}}-\frac{\sigma \beta_{\mathrm{o}}^{2}}{\rho} \mathrm{u}$

$\rho\left(\mathrm{u} \frac{\partial \mathrm{N}}{\partial \mathrm{x}}+\mathrm{v} \frac{\partial \mathrm{N}}{\partial \mathrm{y}}\right)=\frac{\gamma}{\mathrm{j}} \frac{\partial^{2} \mathrm{~N}}{\partial \mathrm{y}^{2}}-\frac{\mathrm{k}_{\mathrm{f}}}{\mathrm{j}}\left(2 \mathrm{~N}+\frac{\partial \mathrm{u}}{\partial \mathrm{y}}\right)$

$\rho c_{p}\left(u \frac{\partial T}{\partial x}+v \frac{\partial T}{\partial y}\right)=k\left(\frac{\partial^{2} T}{\partial y^{2}}\right)+Q\left(T-T_{w}\right)-\frac{\partial q_{r}}{\partial y}$

$\mathrm{u} \frac{\partial \mathrm{C}}{\partial \mathrm{x}}+\mathrm{v} \frac{\partial \mathrm{C}}{\partial \mathrm{y}}=\mathrm{D} \frac{\partial^{2} \mathrm{C}}{\partial \mathrm{y}^{2}}-\frac{\partial}{\partial \mathrm{y}}\left(\mathrm{v}_{\mathrm{t}} \mathrm{c}\right)-\mathrm{R}^{*} \mathrm{c}$

Where $\mathrm{N}$ is the microrotation or angular velocity whose direction of rotation is in the $x$-y-plane, $\mu$ is the viscosity of the fluid, $\square$ is the density, $\mathrm{cp}$ is the specific heat capacity at constant pressure of the fluid, $\mathrm{kf}$ is the thermal conductivity of the fluid, Q is the heat generation/absorption coefficient and $\mathrm{j}, \mathrm{g}$ and $\mathrm{k}$ are the microinertia per unit mass, spin gradient viscosity and vortex viscosity respectively which are assumed to be constant.

The appropriate physical boundary conditions of Eqs (1)-(5) are

$$
\begin{aligned}
& \mathrm{u}(\mathrm{x}, 0)=0 ; v(x, 0)=-v_{o}, N(X, 0)=-n \frac{\partial u}{\partial x} \\
& \mathrm{y} \rightarrow \infty: u(\mathrm{x}, \mathrm{y}) \rightarrow U(x)=a x, v(x, y) \rightarrow 0, N(x, y) \rightarrow 0 \\
& y=0 ; T=T_{w}, c=c_{w} \\
& y \rightarrow \infty: T \rightarrow T_{\infty}, c \rightarrow c_{\infty}
\end{aligned}
$$

Where $n$ is a constant and $0 \leq n \leq 1$. The case $n=1 / 2$ indicates the vanishing of the antisymmetric part of the stress tensor and 
denotes weak concentration of microelements, which will be considered here. Using the transform we have

$\eta=\sqrt{\frac{a}{v}} y, u=a x f^{\prime}(\eta), \quad v=-\sqrt{a v} f(\eta)$

$N=a x \sqrt{\frac{a}{v}} g(\eta), \quad g(\eta)=-\frac{1}{2} f^{\prime \prime}(\eta), \quad \theta=\frac{T-T_{\infty}}{T_{w}-T_{\infty}}$

$\phi=\frac{c-c_{w}}{c_{w}-c_{\infty}}$

After using the transformation (7), for micropolar fluid, there are two equations in which one is for angular velocity or microrotation and physically it is important in micropolar fluid. In this study, we have two equations $f(\eta)$ and $g(\eta)$ which $g(\eta)$ equals to $\frac{-1}{2} f^{\prime \prime}(\eta)[22]$

So Eqns.(2) and (3) reduce to the single equation as Eq. (8a)

$\left(1+\frac{k}{2}\right) f(\eta)+f(\eta) f^{\prime \prime}(\eta)-f^{\prime}(\eta)^{2}-H a^{2} f^{\prime}+1=0$

$\left(1+\frac{4}{3} R\right) \theta^{\prime \prime}(\eta)+\operatorname{Pr} f(\eta) \theta^{\prime}(\eta)+\operatorname{Pr} B \theta(\eta)=0$

$\phi^{\prime \prime}(\eta)+S c\left(f-\tau \theta^{\prime}\right) \phi^{\prime}-S c \tau \phi \theta^{\prime \prime}-\delta S c \phi=0$

Subject to the boundary conditions

$f(0)=A, \quad f^{\prime}(0)=0, \quad f^{\prime}(\infty)=1$

$\theta(0)=1, \quad \theta(\infty)=0$

$(0)=1, \phi(\infty)=0$

Where $\mathrm{K}=\frac{k}{\mu}(>0)$ is the material parameter, $A=\frac{v_{o}}{\sqrt{a v}}$ is the suction parameter and primes denote differentiation with respect to $\eta$. $\operatorname{Pr}=\frac{\mu c_{p}}{k}$ is the Prandtl number and $B=\frac{Q}{\alpha \rho c_{p}}$ is the heat Generation/absorption parameter, $S c=\frac{\mu}{\rho D}$ is the Schmidt number, $\delta=\frac{x R^{*}}{U}$ is the chemical reaction parameter, $\tau=\frac{-k_{f}}{T_{r}}\left(T_{w}-T_{\infty}\right)$ is the thermophoretic parameter, $R=\frac{4 \sigma^{*} T_{\infty}^{3}}{K^{*} K}$ , is the radiation parameter, $H a=\frac{\sigma \beta_{0}^{2}}{\rho \alpha}$ is the magnetic parameter. For micropolar boundary layer flow, the wall skin friction $T_{w}$

$T_{w}=\left[(\mu+k) \frac{\partial u}{\partial y}+k N\right]_{y=0}$

Using $U(x)=a x$ as a characteristic velocity, the skin friction Coefficient, $C_{f}$ can be defined as
$c_{f}=\frac{T_{w}}{\rho U^{2}}$

By using this definition we have

$R e_{x}^{1 / 2}=\left(1+\frac{k}{2}\right) f^{\prime \prime}(0)$

Where $R e_{x}^{1 / 2}=\frac{x U}{v}$ is the local Reynolds Number

The heat transfer from the surface to the fluid is computed by application of Fourier's law

$q=\left[\left(\frac{16 \sigma^{2} T_{\infty}^{3}}{3 k^{*}}\right) \frac{\partial T}{\partial y}\right] y=0$

Introducing the transformed variables, the expression for $q$ becomes and the heat transfer coefficient, in terms of the Nusselt number $\mathrm{N} u$ can be expressed as

$N u=\frac{q}{k\left(T_{w}-T_{\infty}\right) \sqrt{\frac{a}{v}}}$

Then we have $N u=-\theta^{\prime}(0)$

The definition of the local mass flux and the local Sherwood number are respectively given by

$j_{w}=-D\left(\frac{\partial c}{\partial y}\right)_{\mathrm{y}=0}$

$s h_{x}=\frac{j_{w}}{D\left(c_{w}-c_{\infty}\right) \sqrt{\frac{a}{v}}}$

\section{NUMERICAL SOLUTIONS}

Applying the Quasi-linearization technique [ 23] to the nonlinear equation (8a) we obtain as

$(1+K / 2) f^{\prime \prime \prime}+(F) f^{\prime \prime}+\left(-2 F^{\prime}-H^{2}\right) f^{\prime}+F^{\prime \prime} f=F F^{\prime \prime}-F^{\prime} F^{\prime}-1$

Where assumed $F$ is the value of $f$ at $n^{\text {th }}$ iteration and $f$ is at $(\mathrm{n}+1)^{\text {th }}$ iteration. The convergence criterion is fixed as $|\mathrm{F}-\mathrm{f}|$ $<10^{-5}$.

Using an implicit finite difference scheme for the equation $(16),(8 b)$ and (8c), we obtain

$a[i] f[i+2]+b[i] f[i+1]+c[i] f[i]+d[i] f[i-1]=e[I]$

$a_{1}[i] \theta[i+1]+b_{1}[i] \theta[i]+c_{1}[i] \theta[i-1]=0$

$\mathrm{a}_{2}[\mathrm{i}] \phi[\mathrm{i}+1]+\mathrm{b}_{2} \phi[\mathrm{i}]+\mathrm{c}_{2} \phi[\mathrm{i}-1]=0$ 
Where

$$
\begin{aligned}
& \mathrm{a}[\mathrm{i}]=\mathrm{A}[\mathrm{i}], \quad \mathrm{b}[\mathrm{i}]=-3 \mathrm{~A}[\mathrm{i}]+\mathrm{h} \mathrm{B}[\mathrm{i}]+0.5 * \mathrm{~h}^{2} \mathrm{C}[\mathrm{i}] \\
& c[i]=3 A[i]-2 * h B[i]+h^{3} D[i], \\
& \mathrm{d}[\mathrm{i}]=-\mathrm{A}[\mathrm{i}]+\mathrm{h} \mathrm{B}[\mathrm{i}]-0.5 * \mathrm{~h}^{2} \mathrm{C}[\mathrm{i}] \\
& \mathrm{e}[\mathrm{i}]=\mathrm{h}^{3} \mathrm{E}[\mathrm{i}] \\
& \mathrm{a}_{1}=\mathrm{A}_{1}[\mathrm{i}]+0.5 * \mathrm{hB}_{1}[\mathrm{i}], \\
& b_{1}=-2 A_{1}[i]+h^{2} C_{1}[i] \\
& \mathrm{c}_{1}=\mathrm{A}_{1}[\mathrm{i}]-0.5 * \mathrm{~h} \mathrm{~B}_{1}[\mathrm{i}], \\
& \mathrm{a}_{2}=\mathrm{A}_{2}[\mathrm{i}]+0.5 * \mathrm{hB}_{2}[\mathrm{i}] \\
& b_{2}=-2 A_{2}[i]+h * h C_{2}[i] \\
& \mathrm{c}_{2}=\mathrm{A}_{2}[\mathrm{i}]-0.5 * \mathrm{hB}_{2}[\mathrm{i}] \\
& \mathrm{A}[\mathrm{i}]=1+\mathrm{k} / 2, \mathrm{~B}[\mathrm{i}]=\mathrm{F} \text {, } \\
& \mathrm{C}[\mathrm{i}]=-2 \mathrm{~F}^{\prime}-\mathrm{Ha}^{2} \text {, } \\
& D[i]=F^{\prime \prime} E[i]=F F^{\prime}-F^{\prime} F^{\prime}-1 \text {, } \\
& \mathrm{A}_{1}[\mathrm{i}]=1+4 / 3 \mathrm{R}, \\
& \mathrm{B}_{1}[\mathrm{i}]=\operatorname{Prf}[\mathrm{i}] \\
& \mathrm{C}_{1}[\mathrm{i}]=\operatorname{PrB}
\end{aligned}
$$

\section{RESULTS AND DISCUSSION}

Numerical calculations are carried out for different values of dimensionless parameters such as material parameter $\mathrm{K}$, suction parameter A, heat generation/absorption parameter B, Prandtl number $\mathrm{Pr}$, radiation parameter $\mathrm{R}$, thermophoretic parameter $\tau$, chemical reaction parameter $\delta$, Schmidt number $\mathrm{Sc}$ and magnetic number $\mathrm{Ha}$ and a representative set of results is reported in graphs from figures 1 to 18 . These results are obtained to show that the flow field is influenced appreciably by A, K, B, Pr, Sc, R, $\tau, \delta$, Ha.

Figures (1)- (3) represent the dimensionless velocity, temperature and concentration profiles for the values of A respectively. From these figures it is clear that $f^{\prime}(\eta)$ increase with the increase of suction parameter $A$, whereas the temperature and concentration profiles decrease with the increase of suction parameter A. The effect of material parameter $\mathrm{K}$ on velocity, temperature and concentration profiles is displayed in figs. (4) - (6). It is seen that the velocity profile, temperature and concentration profiles decrease with the increase of material parameter $\mathrm{K}$.

The effect of magnetic parameter Ha is shown in figs. (7) - (9) for velocity, temperature and concentration profiles respectively. The velocity profile $f^{\prime}$ decrease with the increase of magnetic parameter $\mathrm{Ha}$, whereas the temperature and concentration profiles increase with the increase of magnetic parameter Ha.

Figures (10)and( 11) show the effect of radiation parameter $\mathrm{R}$ on temperature and concentration profiles respectively. It is noticed that the temperature distribution increase with the increase value of radiation parameter while the concentration profile decrease with the increase of radiation parameter $R$.
The effect of dimensionless Prandtl number Pr on temperature distribution $\theta$ is placed in fig.(12) It can be seen that the temperature profile decrease with the increase of $\operatorname{Pr}$ value. The effect of Prandtl number $\mathrm{Pr}$ is to decrease the concentration profile is observed from fig.(13).

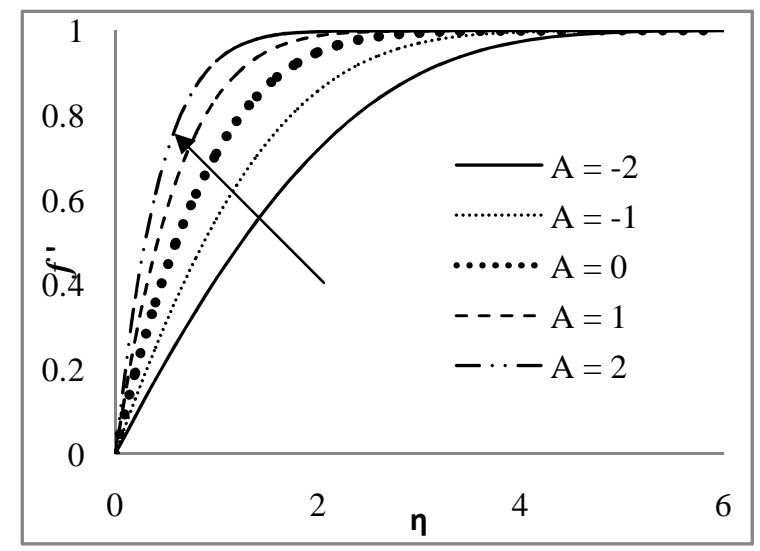

Fig.(1) Effects of Suction parameter A on velocity profile $\mathrm{B}=0.1, \mathrm{~K}=1.0, \mathrm{Pr}=0.72, \mathrm{Sc}=0.66, \mathrm{R}=2.0, \delta=0.4, \tau=1.0$ and $\mathrm{Ha}=0$

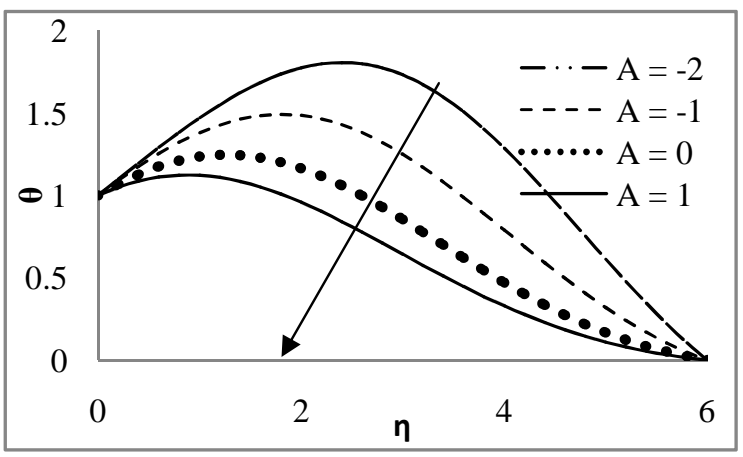

Fig.(2) Effects of Suction parameter A on temperature profile $\mathrm{B}=1, \mathrm{~K}=1.0, \mathrm{Pr}=0.72, \mathrm{Sc}=0.66, \mathrm{R}=2.0, \delta=0.4, \tau=1.0$ and $\mathrm{Ha}=0.5$

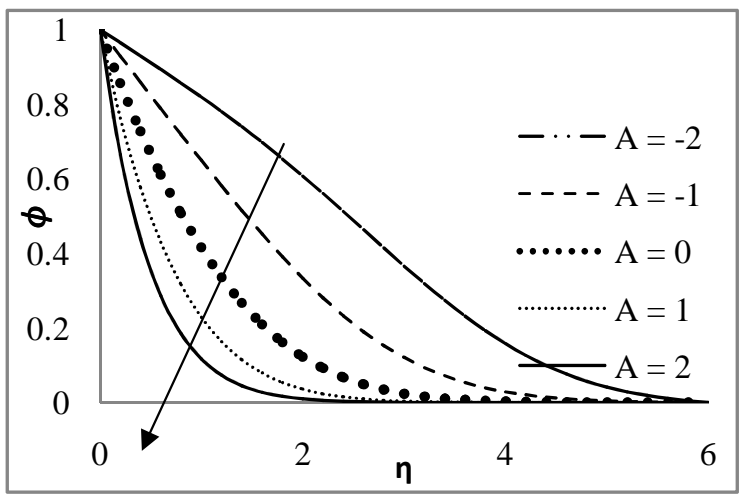

Fig.(3) Effects of Suction parameter A on concentration profile $\mathrm{Sc}=0.66, \mathrm{~K}=1.0, \mathrm{Pr}=0.72, \delta=0.4, \mathrm{~B}=0.1, \tau=1.0, \mathrm{R}=2$ and $\mathrm{Ha}=0.5$ 


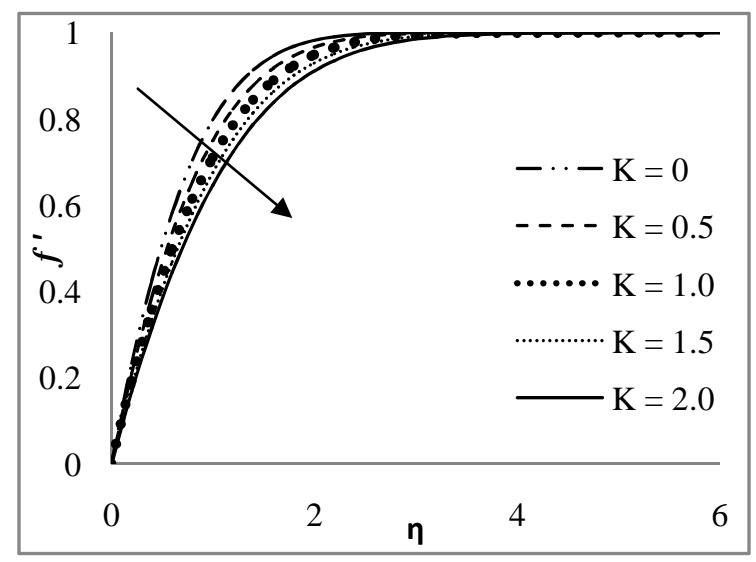

Fig.(4) Effects of material parameter $\mathrm{K}$ on velocity profile $\mathrm{B}=0.1, \mathrm{~A}=0, \mathrm{Pr}=0.72, \mathrm{Sc}=0.66, \mathrm{R}=2.0, \delta=0.4, \tau=1.0$ and $\mathrm{Ha}=0$

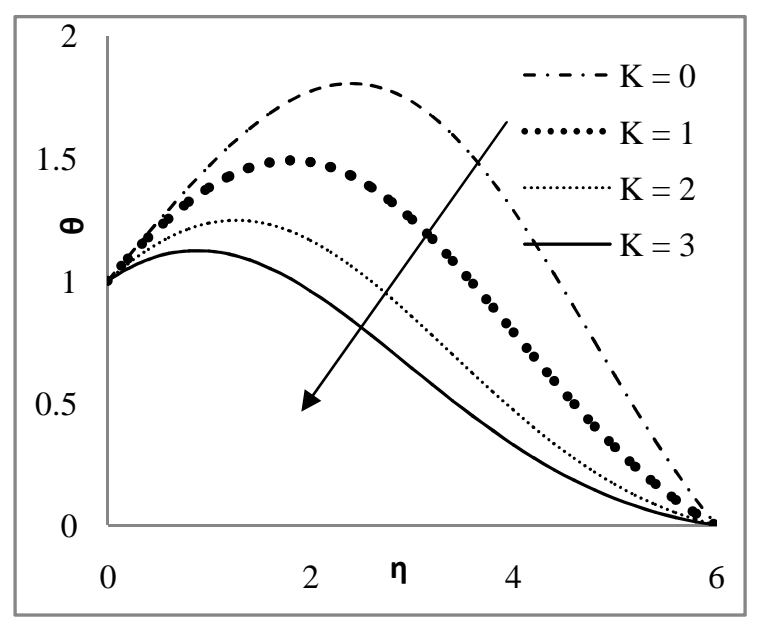

Fig.(5)Effects of material parameter $\mathrm{K}$ on temperature profile $\mathrm{B}=0.1, \mathrm{~A}=0, \mathrm{Pr}=0.72, \mathrm{Sc}=0.66, \mathrm{R}=2.0, \delta=0.4, \tau=1.0$ and $\mathrm{Ha}=0$

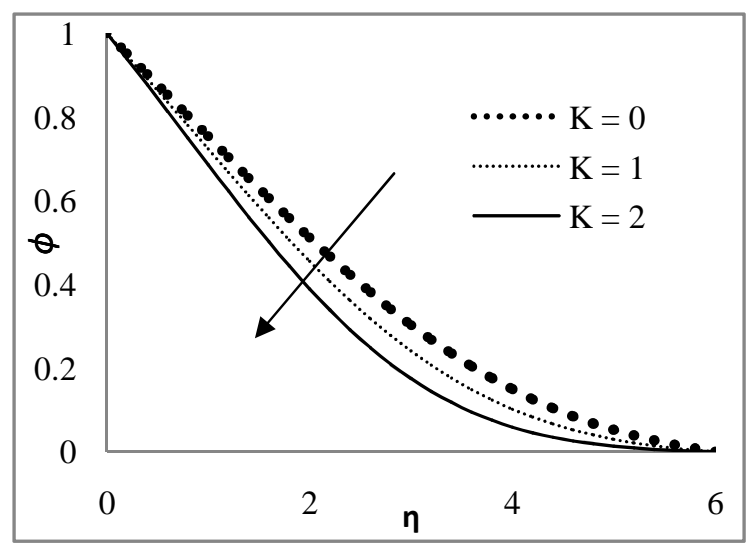

Fig.(6)Effects of material parameter $\mathrm{K}$ on concentration profile $\mathrm{B}=0.1, \mathrm{~A}=0, \mathrm{Pr}=0.72, \mathrm{Sc}=0.66, \mathrm{R}=2.0, \delta=0.4, \tau=1.0$ and $\mathrm{Ha}=0$

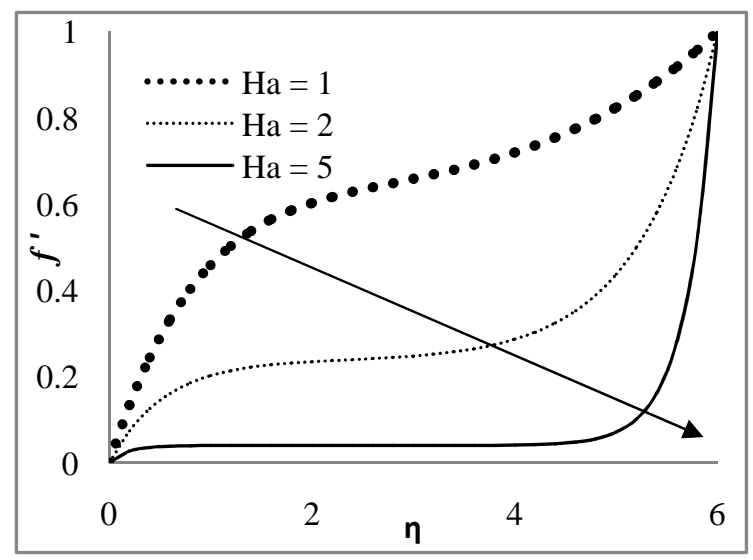

Fig.(7)Effects of magnetic parameter Ha on velocity profile $\mathrm{B}=0.1, \mathrm{~K}=1.0, \mathrm{Pr}=0.72, \mathrm{Sc}=0.66, \mathrm{R}=2.0, \delta=0.4, \tau=1.0$ and $\mathrm{A}=0$

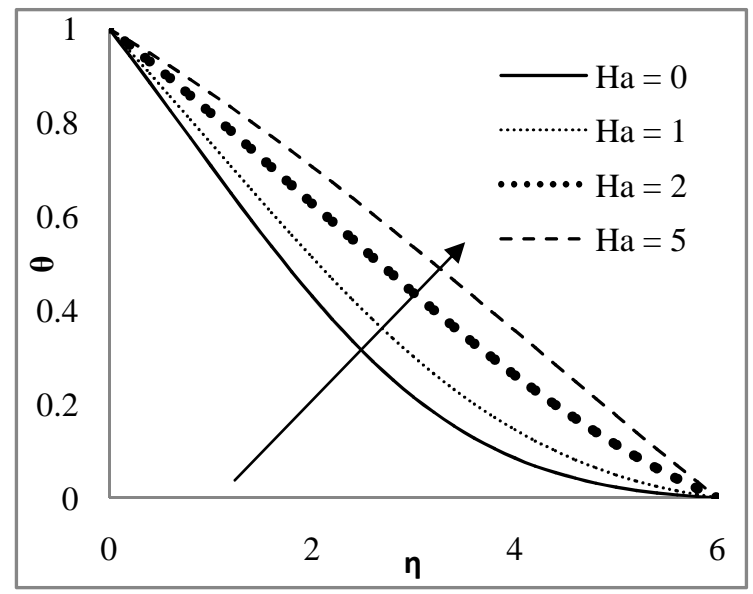

Fig.(8) Effects of Magnetic parameter Ha on temperature profile $\mathrm{A}=0, \mathrm{~K}=1.0, \mathrm{Pr}=0.72, \mathrm{Sc}=0.66, \mathrm{~B}=0.1, \delta=0.4, \tau=1.0$ and $\mathrm{R}=2$

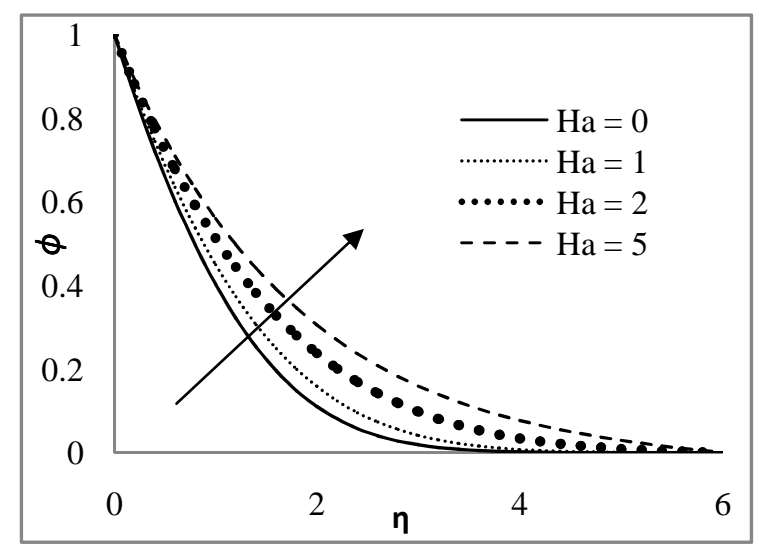

Fig.(9) Effects of Magnetic parameter Ha on concentration profile $\mathrm{Sc}=0.66, \mathrm{~K}=1.0, \mathrm{Pr}=0.72, \delta=0.4, \mathrm{~B}=0.1, \tau=1.0, \mathrm{R}=2$ and $\mathrm{A}=0$ 


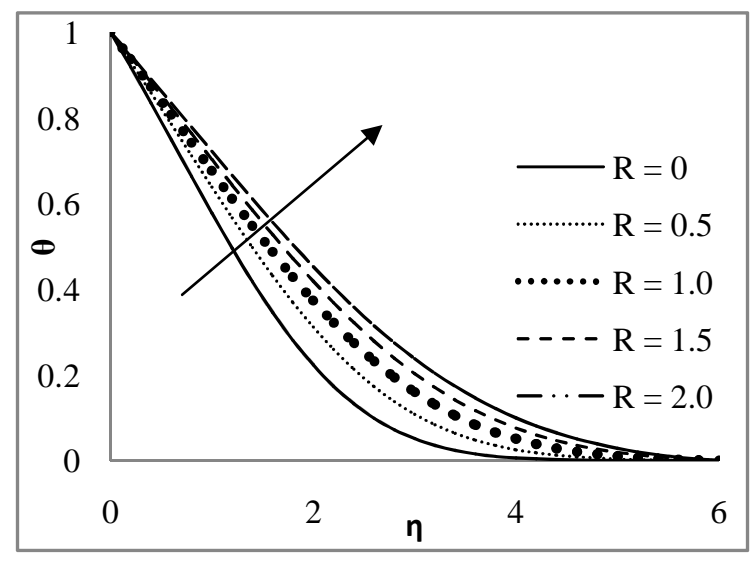

Fig.(10)Effects of Radiation parameter $\mathrm{R}$ on temperature profile $\mathrm{A}=0, \mathrm{~K}=1.0, \mathrm{Pr}=0.72, \mathrm{Sc}=0.66, \mathrm{~B}=0.1, \delta=0.4, \tau=1.0$ and $\mathrm{Ha}=0.5$

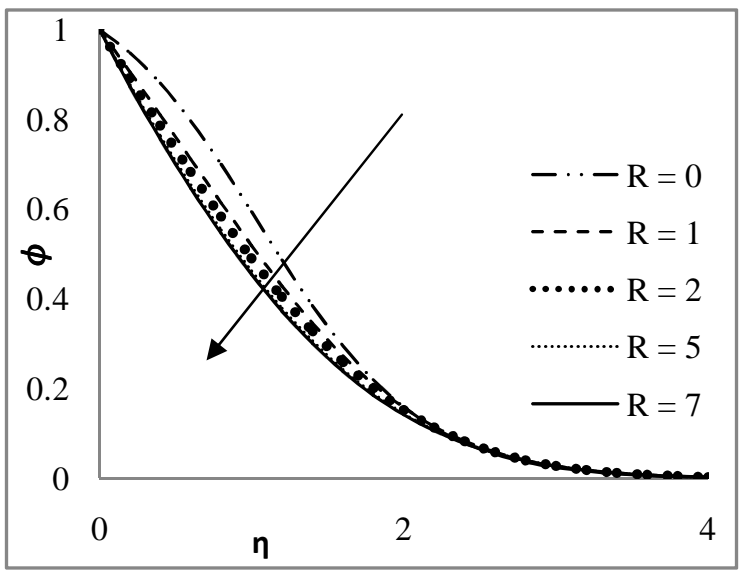

Fig.(11)Effects of Radiation parameter R on concentration profile $\mathrm{A}=0, \mathrm{~K}=1.0, \mathrm{Pr}=0.72, \mathrm{Sc}=0.66$, $\mathrm{B}=1.0, \delta=0.4, \tau=1.0$ and $\mathrm{Ha}=0.5$

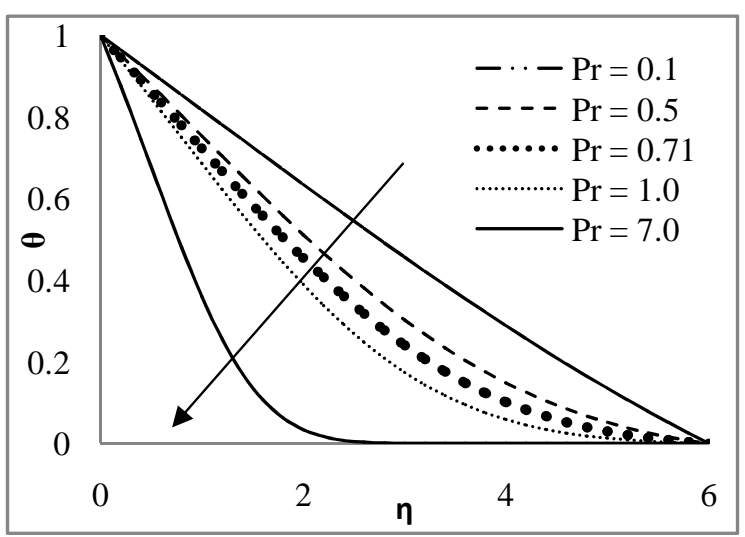

Fig.(12) Effects of Prandtl number Pr on temperature profile $\mathrm{A}=0, \mathrm{~K}=1.0, \mathrm{Ha}=0.5, \mathrm{Sc}=0.66, \mathrm{~B}=0.1, \delta=0.4, \tau=1.0$ and $\mathrm{R}=2$

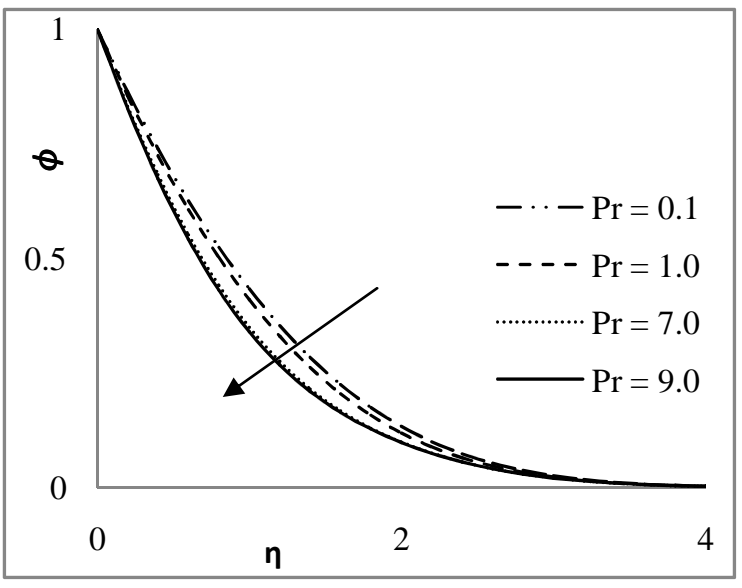

Fig.(13) Effects of Prandtl number Pr on concentration profile $\mathrm{A}=0, \mathrm{~K}=1.0, \mathrm{Ha}=0.5, \mathrm{Sc}=0.66, \mathrm{~B}=0.1, \delta=0.4, \tau=1.0$ and $\mathrm{R}=2$

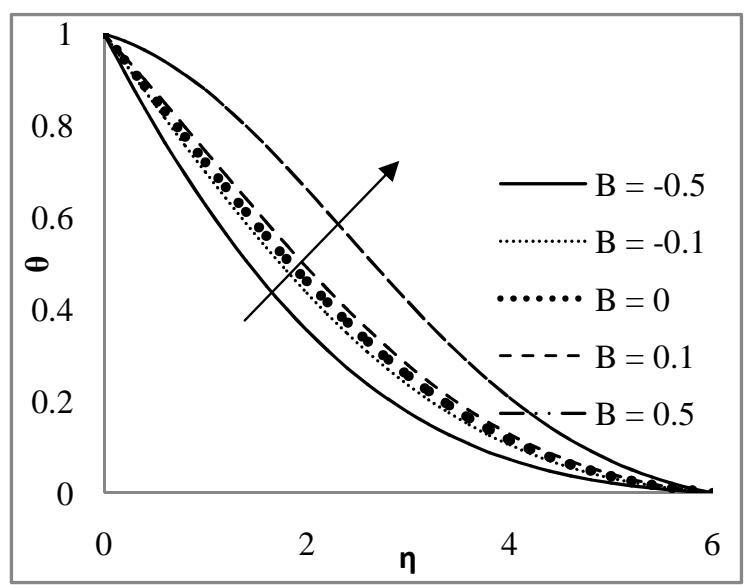

Fig.(14)Effects of heat generation/absorption parameter B temperature profile $\mathrm{A}=0, \mathrm{~K}=1.0, \mathrm{Pr}=0.72, \mathrm{Sc}=0.66$, $\mathrm{R}=2.0, \delta=0.4, \tau=1.0$ and $\mathrm{Ha}=0.5$

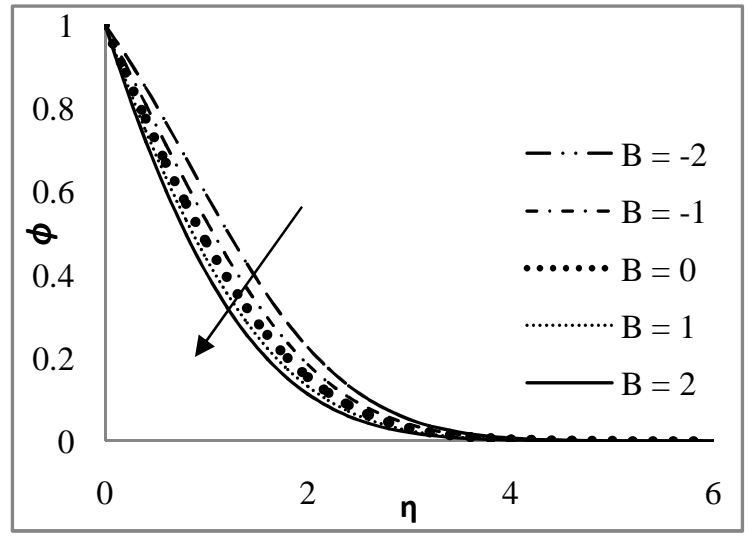

Fig.(15)Effects of heat generation/absorption parameter $B$ on concentration profile $\mathrm{A}=0, \mathrm{~K}=1.0, \mathrm{Pr}=0.72, \mathrm{Sc}=0.66$, $\mathrm{R}=2.0, \delta=0.4, \tau=1.0$ and $\mathrm{Ha}=0.5$ 


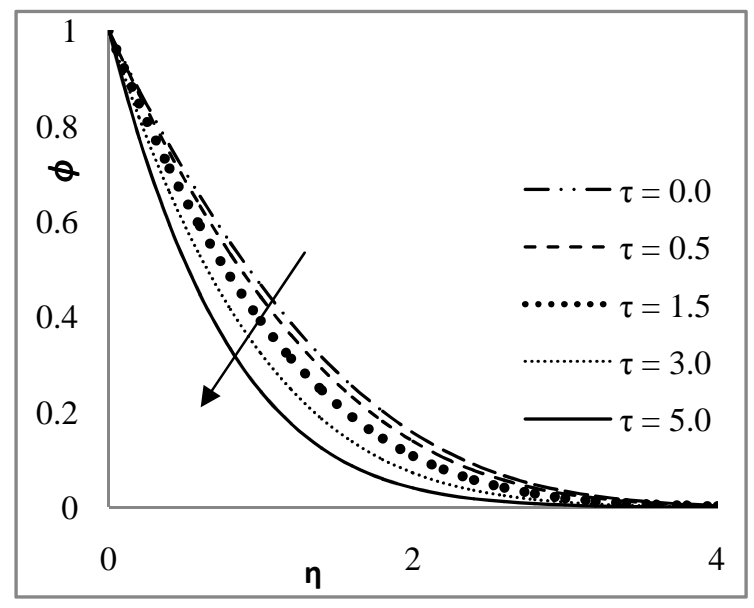

Fig.(16) Effects of thermophoretic parameter $\tau$ on concentration profile $\mathrm{A}=0, \mathrm{~K}=1.0, \operatorname{Pr}=0.72, \mathrm{Sc}=0.66$, $\mathrm{B}=0.1, \delta=0.4, \mathrm{R}=2$ and $\mathrm{Ha}=0.5$

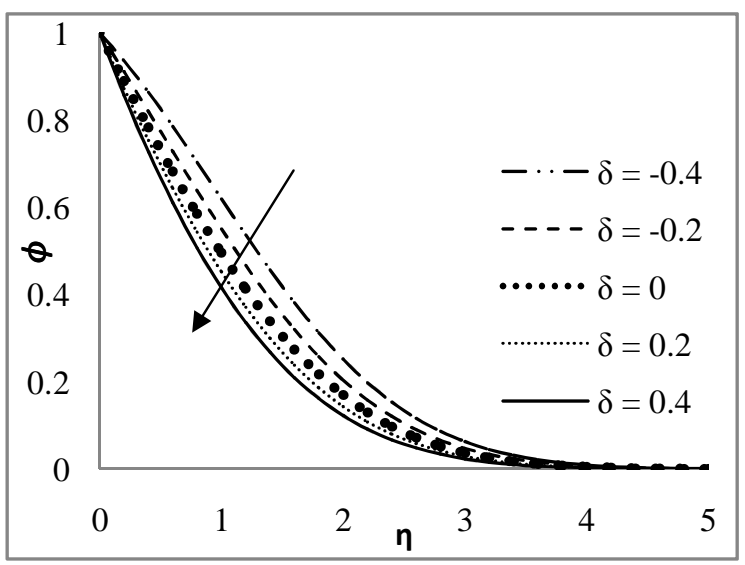

Fig (17)Effects of chemical reaction parameter $\delta$ on concentration profile $\mathrm{A}=0, \mathrm{~K}=1.0, \mathrm{Pr}=0.72, \mathrm{Sc}=0.66, \mathrm{~B}=$ $0.1, \tau=1.0, \mathrm{R}=2$ and $\mathrm{Ha}=0.5$

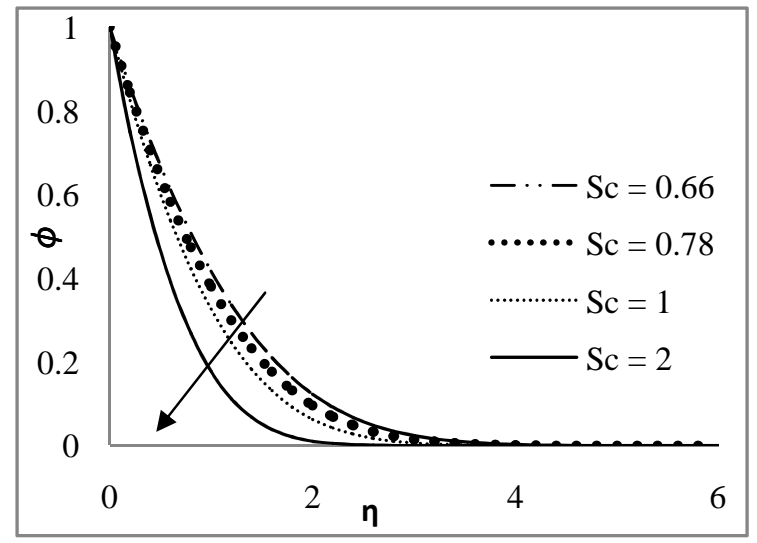

Fig.(18) Effects of Schmidt number Sc on concentration profile $\mathrm{A}=0, \mathrm{~K}=1.0, \mathrm{Pr}=0.72, \delta=0.4, \mathrm{~B}=0.1, \tau=1.0, \mathrm{R}=2$ and $\mathrm{Ha}=0.5$
Table (1) Results of $f^{\prime \prime}(0)$ and - $\theta$ '0) for different values of $\mathrm{K}$ and $\mathrm{A}$ when $\mathrm{B}=0.1$ and $\mathrm{Pr}=0.7$

\begin{tabular}{|l|l|l|l|}
\hline$K$ & $A$ & $f^{\prime \prime}(0)$ & $-\theta^{\prime}(0)$ \\
\hline 0 & 1 & 1.419836 & 0.403682 \\
\hline 0.5 & 1 & -1.201999 & 0.398784 \\
\hline 1.0 & 1 & -1.055735 & 0.39472 \\
\hline 1.5 & 1 & -0.9498 & 0.391236 \\
\hline 2.0 & 1 & -0.86894 & 0.388179 \\
\hline 1 & -2 & 0.378311 & 0.030677 \\
\hline 1 & -1 & 0.519675 & 0.108716 \\
\hline 1 & 0 & 0.737433 & 0.234122 \\
\hline 1 & 1 & 1.055735 & 0.39472 \\
\hline 1 & 2 & 1.494145 & 0.573393 \\
\hline
\end{tabular}

Table (2) Results of - $\theta^{\prime} 0$ ) for different values of $\operatorname{Pr}$ and $B$ when $\mathrm{A}=0$ and $\mathrm{B}=0$

\begin{tabular}{|l|l|l|}
\hline $\operatorname{Pr}$ & $B$ & $-\theta^{\prime}(0)$ \\
\hline 0.05 & 0.1 & 0.171646 \\
\hline 0.1 & 0.1 & 0.17674 \\
\hline 0.5 & 0.1 & 0.219637 \\
\hline 1.0 & 0.1 & 0.271005 \\
\hline 1.5 & 0.1 & 0.314981 \\
\hline 0.7 & -0.1 & 0.303411 \\
\hline 0.7 & 0 & 0.273324 \\
\hline 0.7 & 0.1 & 0.240921 \\
\hline
\end{tabular}

In fig.(14), the effect of heat source/sink parameter $B$ is on the temperature exhibited. From the figure it is noticed that the dimensionless temperature $\theta$ decreases for increasing strength of the heat sink and due to increase of heat source strength the temperature increases. So, the thickness of the boundary layer reduces for increase of heat sink parameter, but it increases with heat source parameter. This result is very much significant for the flow where heat transfer is given prime importance. The concentration profile $\phi$ decrease with the increase of heat generation/absorption parameter B is seen from fig. (15).

Figure(16) is displaced the effect of thermophoretic parameter $\tau$ on concentration profile $\phi$. From the figure it is observed that the influence of thermophoretic parameter $\tau$ is to reduce the concentration profile.

Figure (17) illustrate the variation of concentration profile fpr difference values of $\delta$. The effect of increase of chemical reaction parameter $\delta$ is to decrease concentration profile is noticed from the figure.

Figure (18) shows the effect of Schmidt number Sc on concentration profile. From this figure it is clear that an increase in the Schmidt number results in decrease in the concentration profile. 
Table (1) shown the value of skin friction coefficient $f$ "'(0) and the rate of heat transfer coefficient $-\theta^{\prime}(0)$ for different values of $\mathrm{K}$ and $\mathrm{A}$ for fixed values of $B$ and $P r$. It is observed from the table the skin friction coefficient $f^{\prime \prime}(0)$ decrease with the increase of $K$ whereas the value of $f^{\prime \prime}(0)$ increase with the increase of $A$. It can also be seen that the heat transfer coefficient $-\theta^{\prime}(0)$ decrease with the increase of $K$ value. The heat transfer coefficient $-\theta^{\prime}(0)$ increase with the increase of $A$ value from -2 to 1 .

Table (2) shown that heat transfer coefficient $-\theta^{\prime}(0)$ for different values of Prandtl number $\mathrm{Pr}$ and heat generation/absorption parameter $B$. It is observed from the table heat transfer coefficient $-\theta^{\prime}(0)$ value increase with the increase of $\operatorname{Pr}$ value whereas it decreases with the increase of $B$ value from -0.1 to 0.1

\section{CONCLUSIONS}

The problem of steady, laminar, two dimensional stagnation point flow of an incompressible flow non Newtonian micropolar fluid with uniform suction/blowing, heat generation/absorption, radiation, thermophoresis and chemical reaction under the influence of magnetic field. The effects of the various dimensionless parameters are investigated.

1.The velocity profile is decreased with the increase of material parameter $K$ and magnetic parameter $\mathrm{Ha}$ suction parameter $A$.

2.The temperature profile increases with the increase of magnetic parameter $\mathrm{Ha}$, heat generation/absorption $\mathrm{B}$ and radiation parameter $R$. The temperature profile decreases with the increase of suction parameter $A$, material parameter $K$ and Prandtl number $P r$.

3.The concentration profile increase with the increase of magnetic parameter $\mathrm{Ha}$ whereas the concentration profiles decreases with increase of material parameter $K$, suction parameter $A$, Prandtl number $P r$, thermophoretic parameter $\tau$, chemical reaction parameter $\delta$, heat generation/absorption parameter $B$, radiation parameter $R$ and Schmidt number $S c$.

\section{REFERENCES}

[1]. Eringen A.C. Theory of micropolar fluids, J. Math. Mech., (1966), Vol. 16, pp. 1-18.

[2]. Eringen A.C. Theory of thermo micro fluids, J. Math. Anal. Appl., (1972), Vol. 38, pp. 480-496.

[3] Ariman T, Turk M A, Sylvester N D. Microcontinuum fluid mechanics-are view. Int J Engng Sci, 11 (1973)905-930

[4] Gorla R S R. Mixed convection in a micropolar fluid from a vertical surface with uniform heat flux. Int J Engng Sci, 30 (1992)349-358.

[5] Rees D A S, Pop I. Free convection boundary layer flow of a micropolar fluid from a vertical flat plate. IMAJ Appl Math, 61 (1998)179-197.
[6] Singh Ajay Kumar. Numerical solution of unsteady free convection flow of an incompressible micropolar fluid past an infinite vertical plate with temperature gradient dependent heat source J Energy Heat and Mass Transfer, 24 (2002)185-194.

[7]Cussler E L. Diffusion mass transfer in fluid systems 2nd Ed. Cambridge: Cambridge University Press. 1998.

[8] Das U N, Deka R K, Soundalgekar V M. Effects of mass transfer on flow past an impulsively started infinite vertical plate with constant heat flux and chemical reactionForschung im Ingenieurwesen Engineering Research, 60 (1994)284-287.

[9] Muthucumarswamy R, Ganesan P. First order chemicalreaction on flow past an impulsively started vertical plate $\mathrm{h}$

[10] Muthucumarswamy R. Effects of a chemical reactionon moving isothermal vertical surface with suction. Acta Mech, 155 (2002)65-70.

[11] Hinds. W.C, Aerosol Technology: Properties, Behavior, and Measurement of Airborne Particles, John Wiley and Sons, New York, 1982.

[12]Tsai. C.J, Lin. J.S, Shankar. I, Aggarwal.G, Chen.D.R., Thermophoretic deposition of particles in laminar and turbulent tube flows, Aerosol Sci. Technol. 38 (2004) 131139.

[13] Bakier.A.Y, Mansour. M.A, Combined magnetic field and thermophoresis particle deposition in free convection boundary layer from a vertical flat plate embedded in a porous medium, Int. J. Thermal. Sci. 11 (2007) 65-74.

[14]. Balaram $M$ and Sastry V.U.KMicropolar free convectionflow, Int. J. Heat Mass Transf., . (1973), Vol.16, pp.437-440.

[15]. Agarwal R.S. Dhanapal C. Numerical solution of free convectionmicropolarfluid flow between two parallel porous vertical plates, Int. J. Eng. Sci., (1988), Vol. 26, pp.12471255 .

[16]. Srinivasacharya D., Ramana Murthy J.V.andVenugopalam D. Unsteady stokes flow of micropolarfluidbetweentwoparallel porous plates, Int. J. Eng. Sci., (2001), Vol. 39, pp. 1557-1563.

[17]. Gorla R.S.R., Takhar H.S., and Slaout,A Magnetohydrodynamic free convection boundary layer flow of a thermomicropolar fluid over a vertical plate, Int. J. Eng. Sci., (1998), Vol. 36, pp. 315-327.

[18]. El-Hakiem M.A., Mohammadein A.A., El-Kabeir S.M.M. and Gorla R.S.R. Joule heating effects on magnetohydrodynamic free convection flow of a micropolar fluid, Int. Commun.Heat Mass Transf. (1999), Vol. 26, pp. 219-227.

[19]. Helmy K.A., Idriss H.F. and Kassem S.E. (2002), MHD free convection flow of a micropolar fluid past a vertical porous plate, Can. J. Phys./Rev. Can. Phys. Vol.80, pp. 16611673.

[20]. Bhargara R., Kumar L. and Takhar H.S. (2003), Numerical solution of free convection MHD micropolar fluid flow between two parallel porous vertical plates, Int. J. Eng. Sci., Vol.41, pp.123-136. 
[21] R. A. Mohamed1 et al. Heat and mass transfer analysis on the flow of non-Newtonian micropolar fluid with uniform suction/blowing, heat generation, chemical reaction and Thermophoresis effects. American Academic \& Scholarly Research Journal, Special Issue - January 2012

[22] Ziabakhsh. Z, Domairry. G, Bararnia. H. Analytical solution of non-Newtonian micropolar fluid flow with uniform suction/ blowing and heat generation. J. Taiwan Institute of Chemical Engineers 40 (2009) 443-451

[23] Bellman,R.B., and R.E.Kalaba(1965),Quasi-linearization and Non-Linear boundary value problem, Elsevier, Newyork. 